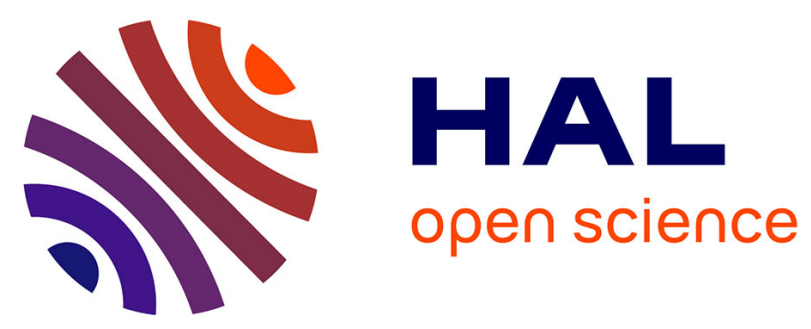

\title{
MISE AU POINT D'UNE TECHNIQUE D'INTENSIMÉTRIE POLAIRE : PERSPECTIVES D'UTILISATION
}

J. Migneron, P. Lemieux, M. Levavasseur

\section{- To cite this version:}

J. Migneron, P. Lemieux, M. Levavasseur. MISE AU POINT D'UNE TECHNIQUE D'INTENSIMÉTRIE POLAIRE: PERSPECTIVES D'UTILISATION. Journal de Physique IV Proceedings, 1992, 02 (C1), pp.C1-457-C1-460. 10.1051/jp4:1992198 . jpa-00251270

\section{HAL Id: jpa-00251270 \\ https://hal.science/jpa-00251270}

Submitted on 1 Jan 1992

HAL is a multi-disciplinary open access archive for the deposit and dissemination of scientific research documents, whether they are published or not. The documents may come from teaching and research institutions in France or abroad, or from public or private research centers.
L'archive ouverte pluridisciplinaire HAL, est destinée au dépôt et à la diffusion de documents scientifiques de niveau recherche, publiés ou non, émanant des établissements d'enseignement et de recherche français ou étrangers, des laboratoires publics ou privés. 


\title{
MISE AU POINT D'UNE TECHNIQUE D'INTENSIMÉTRIE POLAIRE : PERSPECTIVES D'UTILISATION
}

\author{
J.G. MIGNERON, P. LEMIEUX et M. LEVAVASSEUR \\ Laboratoire d'Acoustique de l'Université Laval, CRAD 1636, Pavillon Félix-Antoine Savard, Québec \\ G1K 7P4, Canada
}

\begin{abstract}
A robotic apparatus was designed and developed to take rapidly polar sound intensity measurements. This technique has a double dimension: it entails the expression of the relationship between the polar information and the standard acoustical parameters (pressure, simple vectorial composition of the flux line or complexe temporal and vectorial composition), and secondly, the robotic handling of the sound intensity probe. The robot can be interfaced, through a PS/2 computer, to dual channel third octave real time analyser or to double FFT analyser. It can use an orthogonal routine, allowing a fast reading of the three vectorial components in a stationary acoustical mode, and it can be programmed to search automatically the maximum intensity level for the determination of the flux line or, in near field, with the aim of reactive intensity. In addition to conducting polar sound intensity experiments in stationary regime and in laboratory conditions, potential utilization of this technique was developed for complex industrial acoustical fields or for architectural acoustics.
\end{abstract}

\section{RELEVÉ POLAIRE DE L'INTENSITÉ}

Pourquoi effectuer des relevés polaires? Pour une incidence sonore de $90^{\circ}$ (ou $270^{\circ}$ ) par rapport à l'axe de la sonde intensimétrique, il n'existe théoriquement aucune composante de l'intensité. Or, on constate, en pratique, que la sonde enregistre, sous cet angle d'incidence, des valeurs plus ou moins fantaisistes (point d'ailleurs en partie reconnu par les fabricants [1]). Cette situation est facilement identifiable lors de la lecture d'un relevé polaire. Par contre, avec un relevé selon trois composantes perpendiculaires (principe des sondes tridimensionnelles avec trois doublets de microphones [2]), il suffit qu'on ait l'une de ces composantes confondue avec cette incidence critique pour que le vecteur intensité ainsi composé soit erroné. On peut d'ailleurs ajouter, que dans la mesure ou le dispositif robotique est suffisamment rapide, il peut remplacer aisément un capteur à six microphones, ceci à l'aide de trois rotations consécutives.

Les relevés polaires visent d'abord à exploiter au mieux les indications de directivité fournies par la nature vectorielle de l'intensité. En déterminant complètement cette dernière (norme et direction), il est possible de localiser la provenance exacte du champ acoustique étudié, voire même d'estimer sa contribution en énergie dans une direction donnée. Ces informations sont requises, par exemple, afin de contrôler efficacement le rayonnement d'une source, qu'on désire la renforcer (en acoustique architecturale), ou bien la réduire (en acoustique industrielle). La technique peut s'avérer également utile pour localiser les zones de propagation, ou bien d'ombre, d'un ensemble de sources. De même, elle peut permettre un bilan spatiotemporel autour d'un point de réception déterminé (tel qu'un poste de travail en milieu industriel).

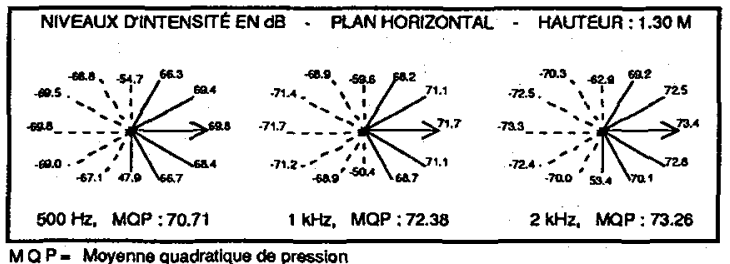

FIGURE No1: Présentation d'un relevé polaire

Un relevé polaire fournit uniquement les composantes du vecteur intensité, à l'aide des niveaux d'intensité mesurés dans l'axe de la sonde, ceci en effectuant, par exemple, une lecture après chaque rotation de $30^{\circ}$. Comme le montre la Figure no 1 , la flèche qui apparaît sur le relevé indique une direction de 
référence choisie par l'utilisateur. La rotation se fait toujours dans le sens trigonométrique: un trait plein indique une intensité "positive" et un trait en pointillés indique une intensité "négative".

RASMUSSEN explique que pour décrire un champ vectoriel, on a besoin d'explorer le champ dans toutes les directions, de manière à trouver la lecture maximale [3]. Lorsqu'on effectue un relevé dans un plan considéré, il n'est pas toujours évident, que l'un des axes de mesure soit rigoureusement confondu avec la direction de propagation. En pratique, il faut considérer comme direction de propagation, dans un plan déterminé, la direction pour laquelle le plus grand niveau d'intensité est enregistré, les autres vecteurs étant considérés comme ses composantes. Avec la technique polaire, le vecteur intensité sera toujours localisé dans un cône dont l'angle au sommet n'excèdera pas le pas angulaire de mesure.

\section{RÉAlisation TECHNIQUE}

Les grandes lignes du modèle de robot adapté à la manipulation polaire de la sonde intensimétrique ont été définies dans la perspective des besoins du Laboratoire d'acoustique et des projets de recherche en cours. Après discussion avec l'organisme constructeur (département de robotique du Collège de LévisLauzon), le concept final est représenté sur la Figure no 2 .

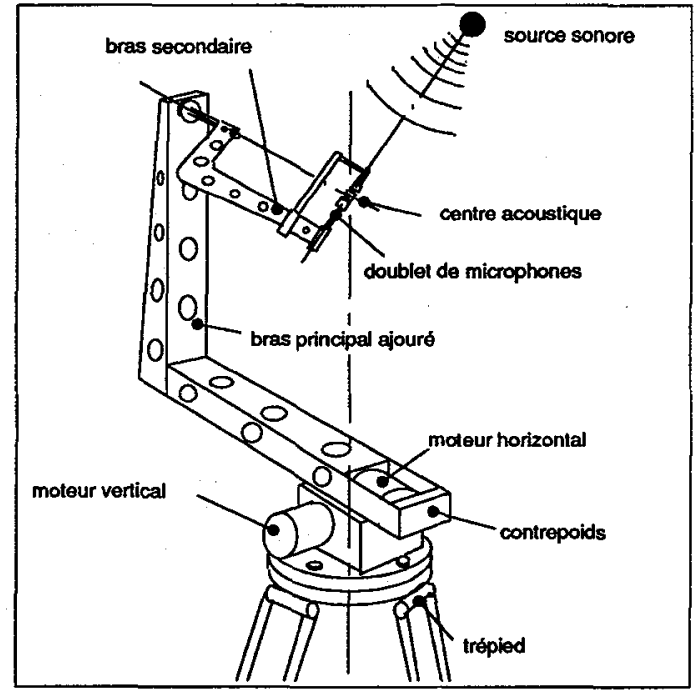

FIGURE № 2: Croquis du robot manipulateur pour la sonde intensimétrique.

Le robot est construit autour de deux axes de rotation, perpendiculaires entre eux, leur intersection étant placée juste au centre acoustique du doublet de microphones de la sonde. Le moteur du bras horizontal est ramené vers la base, afin d'agir comme contrepoids, et le réducteur vertical, destiné à absorber la plus forte inertie, est constitué d'un engrenage avec une vis sans fin. Finalement, le robot repose sur une masse d'inertie démontable, pour faciliter son transport lors des mesures intensimétriques. Le dégagement vertical est d'environ $500 \mathrm{~mm}$, sous la sonde intensimétrique, et le dégagement latéral de $250 \mathrm{~mm}$, de façon à ne pas affecter le champ acoustique à mesurer. Toutes les pièces constituant les bras sont percées, pour la même raison, de trous de différents diamètres.

Les principaux éléments du cahier des charges ont été les suivants: angles de rotation normaux, suivant les deux axes, $5^{\circ}, 10^{\circ}, 15^{\circ}, 30^{\circ}$ ou $90^{\circ}$, avec la possibilité de programmer toute autre valeur; couverture complète des $360^{\circ}$, par une cheminement alternatif, vers la gauche puis vers la droite (pour éviter de mêler les câbles); temps moyen de déplacement entre deux positions de mesure: $1 \mathrm{sec}$.; précision de la position du centre acoustique de la sonde, dans toutes les directions de l'espace, $1 \mathrm{~mm}$. Le dispositif robotique est piloté par l'intermédiaire d'un ordinateur IBM PS/2. Les deux préamplificateurs de microphones viennent se raccorder à un analyseur bicanal en temps réel, au tiers d'octave, de Brüel \& Kjaer, modèle 2133, ce qui constitue une chaîne de mesure complètement automatisée. L'analyseur est en effet interfacé également au même ordinateur, via un bus IEEE-488. La programmation de base comporte trois déplacements de référence, soit le plan horizontal (12 vecteurs), le plan vertical défini par sa positon angulaire, de $-60^{\circ}$ à - $60^{\circ} \mathrm{du}$ plan horizontal (11 vecteurs), la calotte sphérique supérieure, jusqu'à - $60^{\circ}$ du plan horizontal (61 vecteurs), plus une procédure orthogonale rapide. 
Après quelques mois d'expérimentation, il est déjà acquis que, dans un champ acoustique stationnaire, le robot peut chercher très rapidemment, par optimisations successives, la position exacte de la ligne de flux et procéder ainsi à la détermination du vecteur intensité vrai. C'est d'ailleurs cette procédure itérative, par pas angulaire de $5^{\circ}$, qui est illustrée dans la Figure $n^{2} 3$.

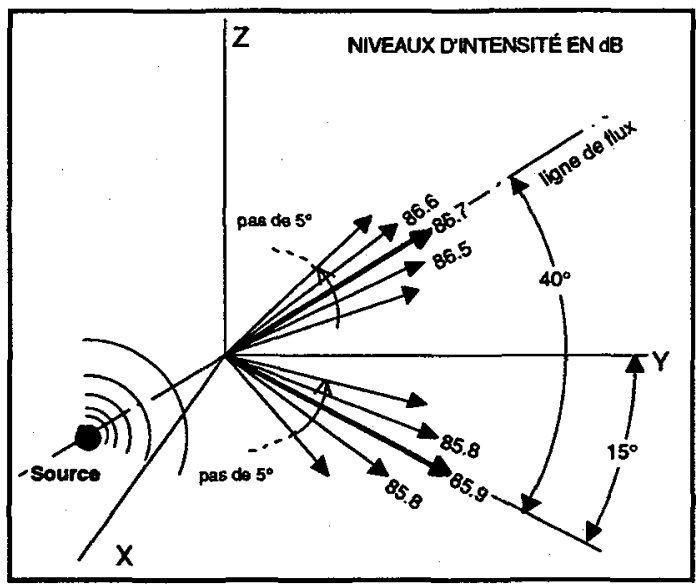

FIGURE № 3: Recherche automatique de la ligne de flux.

\section{DIFFÉRENTS MODES D'utilisation}

Dans des conditions de laboratoire ou en acoustique des salles, il est possible d'utiliser des sources fixes, de radiation constante dans le temps (en intensité et en directivité), cette situation corrrespond à un champ acoustique stationnaire (même diffus) [4]. C'est certainement dans ces conditions que le robot peut être utilisé le plus rapidement, en réduisant à son minimum le temps d'acquisition des niveaux d'intensité.

Pour certaines situations industrielles, aux perturbations de l'air près, il peut en être exactement de même. Néanmoins, il ne s'agit pas d'un cas général; la plupart du temps, les sources sont variables en durée d'émission, composition spectrale, puissance, directivité, voire même, position géométrique dans l'espace. Les champs acoustiques industriels sont donc aléatoires, ils doivent être traités idéalement sur une base statistique. Au plan pratique, il convient, en particulier, d'augmenter sensiblement le temps de moyennage de l'analyseur bicanal $[5,6]$.

A titre d'exemple, la Figure no4 montre deux cas réels d'application, l'un dans l'industrie, pour un poste de travail situé entre deux sources de bruit, et l'autre en acoustique architecturale. Dans ce dernier cas, il s'agissait d'identifier la part de l'énergie réfléchie par le mur d'arrière-scène; la source sonore omnidirectionnelle avait été placée au centre de la conque d'orchestre (Salle Louis Fréchette du GrandThêâtre de Québec).

\section{PERSPECTIVES de DÉVELOPPEMENT}

L'optimisation de la technique d'acquisition automatique de l'intensimétrie polaire offre des perspectives de développement intéressantes, dont plusieurs aspects dépassent largement la seule dimension métrologique. Trois aspects font actuellement l'objet de travaux de recherche complémentaires, ce sont les suivants:

- la comparaison entre la procédure de mesure triaxiale orthogonale rapide et l'emploi d'une sonde intensimétrique classique à six microphones: dans bien des situations, il est fort possible que la procédure d'acquisition robotisée puisse être aussi précise et presque aussi rapide que l'emploi simultané de trois analyseurs bicanaux ou bien d'un mutiplexeur électronique;

- l'optimisation de la procédure de recherche automatique de la ligne de flux: il s'agit là d'un développement prometteur au plan scientifique, cette procédure est susceptible d'intéresser les fírmes d'instrumentation, car c'est un bon moyen pour mesurer simplement et directement l'intensité propagative vraie (en fournissant son amplitude et ses coordonnées angulaires);

- et, un dernier aspect, important pour le contrôle du bruit industriel, soit l'optimisation de l'acquisition statistique d'un champ acoustique variable en fonction du temps: il s'agit ici d'arriver à une spatialisation de la dose de bruit autour d'un poste de travail donné. La dose conventionnelle 
peut ainsi être intégrée dans toutes les directions de l'espace, pendant toute la durée de la période de travail ou d'exposition au bruit.
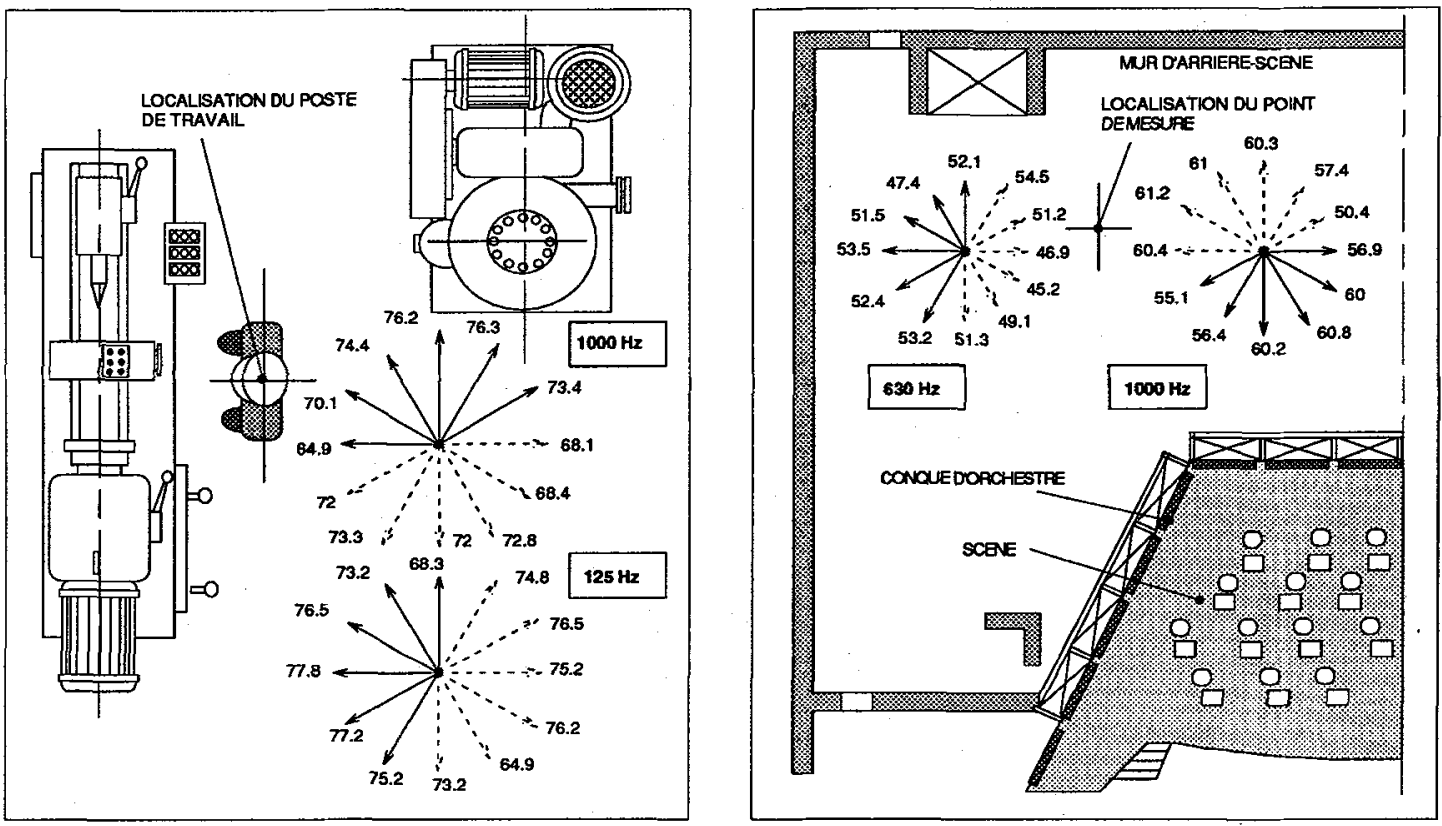

FIGURE № 4: Exemples d'applications industrielle et architecturale de la technique des relevés polaires.

Une fois optimisé le temps de moyennage de l'analyseur, le logiciel à développer pourra donner en même temps une distribution spatiale des fréquences et une distribution globale en $\mathrm{dB}(\mathrm{A})$, cette dernière représentant la distribution spatiale de la dose de bruit. Ce type de relevé sera particulièrement intéressant pour les postes de travail fixes, en vue, notamment, de l'élaboration d'enceintes de protection acoustiques partielles $[7,8]$.

\section{RÉFÉRENCES}

[1] BRUEL \& KJAER, Intensité Acoustique (opuscule), publication of Brüel \& Kjaer, janvier 1987.

[5] MIGNERON, Jean-Gabriel, M. ASSELINEAU, R. WOODCOCK, A. ESTEVE, P. JACQUES et R. MARY, "Utilisation de lintensimétrie pour l'analyse du champ sonore autour de vingt postes de travail sur les machines à papier de la compagnie Kruger à Trois-Rivières", 387 p., projet N/D RS-85-43, CRAD/IRSST, juin 1987.

[6] MIGNERON, Jean-Gabriel, M. ASSELINEAU, R. WOODCOCK et A. ESTEVE, "Utilisation de l'intensimétrie pour l'analyse du champ sonore autour de deux postes de travail sur la machine à papier de la compagnie Donohue à Amos", 79 p., projet N/D RS-85-43, CRAD/IRSST, mars 1987.

[7] MIGNERON, Jean-Gabriel, "Étude intensimétrique du champ sonore autour de différents postes de travail, modélisation de la diffraction et de la protection résultant d'enceintes partielles", pp.56-61, in Semaine Can. d'Acoust., Calgary, octobre 1987.

[8] MIGNERON, Jean-Gabriel, P. Lemieux et M. Levavasseur, "Utilisation de l'intensimétrie pour l'analyse du champs sonore autour d'un poste de travail et pour la mise au point d'enceintes partielles de protection contre le bruit", 83p., CRAD/IRSST, juin 1990.

[4] PÉPIN, H., Caractérisation du champ diffus dans les locaux par intensimérrie, colloque de physique, premier congrès français d'acoustique 1990, colloque C2, supplément au No2, tome 51, février 1990, 1085-1088.

[3] RASMUSSEN, G., Measurements of Vector Fields, 2nd International Congress on Acoustic Intensity, CETIM, Senlis (France), 23 au 26 septembre 1985, recueil de conférences 53-58.

[2] WASMER, J., "Sonde intensimétrique pour l'analyse intensimétrique en temps réel à trois dimensions", pp.927-930, in Premier Congrès Français d'Acoust., Vol.2, Journ. de Physique, Tome 51, février 1990. 